\title{
THE DETERMINATION OF LIMITING PRESSURE IN SIMULTANEOUS ELONGATION AND INFLATION OF NONLINEAR ELASTIC TUBES
}

\author{
Robert Benedict, Alan Wineman and Wei H. Yang \\ Department of Applied Mechanics and Engineering Science, The University of Michigan, Ann Arbor, \\ MI 48109, U.S.A.
}

(Received 12 September 1977; in revised form 5 July 1978)

\begin{abstract}
A nonlinear elastic tubular membrane bonded at its ends to rigid plates is subjected to internal pressure and elongation. For a fixed elongation, pressure initially increases with radius, reaches a local maximum and then decreases. The purpose of this work is to determine this limiting pressure for each prescribed elongation. The usual boundary value problem formulation is such that a two dimensional search must be conducted. This can be computationally very costly. A method is presented which reduces the determination of the limiting pressure to a one-dimensional search. A numerical example is presented.
\end{abstract}

\section{INTRODUCTION}

An interesting phenomenon in nonlinear elasticity arises in the problem of the inflation of a spherical membrane by internal pressure. In studying this problem for an incompressible Mooney model, Green and Shield[1] showed that the inflating pressure $p$ need not montonically increase with deformed radius $r$. In particular, $p$ can increase montonically. to a local maximum, decrease to a local minimum and then increase once more. This local maximum represents a limiting pressure. If a higher internal pressure is applied, the membrane will either respond dynamically or assume a much larger equilibrium state.

The same phenomenon appears in other membrane problems, such as the inflation by lateral pressure of a flat circular membrane clamped along a boundary [2] or the inflation of a torus by internal pressure [3]. In each of these cases, the pressure has a local maximum when considered as a function of an appropriate deformation parameter. Again for the reason discussed above, it is useful to know the magnitude and deformation at this local maximum.

In the above examples there is only one load parameter, the internal pressure. Now consider a nonlinear, elastic, cylindrical membrane which is bonded at its ends to rigid plates. The membrane is to be subjected to simultaneous inflation and elongation. Two load parameters must now be specified, the internal pressure and either the elongation or force applied to the end plates.

This problem was first solved analytically by Kydoniefs and Spencer [4] for the special case of zero end forces. They presented deformed profiles for a number of internal pressures. Their results indicate that the relation between pressure and deformed radius at mid-length has a local maximum. However, this case is governed by only one load parameter, the pressure. The present work is concerned with determining the local maximum in the more general case, when two parameters are varied.

For the cases of the pressurized spherical and toroidal membranes, and the clamped membrane, it is possible to construct the pressure-geometry relation by an inverse procedure. The problem formulations are such that the local maximum can be found by a one parameter search. On the other hand, the formulation of the tube problem is such that a two parameter search must be conducted. Since this can be very time consuming, often prohibitive in computing cost, an alternate method is desirable The one presented here is an optimization scheme using a projected gradient. By means of this method, the search for the maximum pressure is confined to a path (a one dimensional space) instead of a two dimensional space, allowing a significant saving in computer time to be realized. It is applied to the tube problem to obtain results for this interesting case as well as to demonstrate the method for more complicated problems.

The problem is formulated in Section 2. A numerical method for solving the boundary value problem for any prescribed pressure and either length or force is outlined in Section 3. The 
projected gradient optimization scheme is developed in Section 4. Results for a specific kind of elastic material are presented in Section 5.

\section{FORMULATION}

The midsurface of the undeformed membrane is a circular cylinder of radius $a$ and length $2 L_{0}$. The membrane has uniform initial thickness $h_{0}$. The ends of the tube are bonded to rigid circular end plates of radius $a$. These end plates are considered attached to a loading device in that either prescribed end forces or elongation can be applied.

Placing cylindrical coordinates with origin at mid-height, the undeformed membrane consists of particles at $(a, \theta, z), 0 \leq \theta \leq 2 \pi,-L_{0} \leq z \leq L_{0}$. A typical particle moves to $(\rho(z), \theta, z(z))$ (see Fig. 1). Assuming axial symmetry, principal directions of stretch and stress are known a priori to be tangent to the membrane in the meridional (1) and circumferential (2) directions and normal to the surface. The stretch ratios are given by, respectively,

$$
\lambda_{1}=\left[\left(\rho^{\prime}\right)^{2}+\left(z^{\prime}\right)^{2}\right]^{1 / 2}, \quad \lambda_{2}=\rho / a, \quad \lambda_{3}=\left(\lambda_{1} \lambda_{2}\right)^{-1},
$$

where ()$^{\prime}=\mathrm{d}() / \mathrm{d} z$ and $\lambda_{3}$ is determined by the incompressibility condition. If $\sigma_{\alpha}$ denotes a principal stress, its stress resultant per unit length in the membrane surface is $T_{\alpha}=h_{0} \lambda_{3} \sigma_{\alpha}$, $(\alpha=1,2)$. Introduce non-dimensional coordinates $\bar{z}=z / a, \bar{\rho}=\rho / a$ and $\bar{z}=\xi / a$ and stress $\bar{\sigma}_{\alpha}=\sigma_{\alpha} / C_{0}$, where $C_{0}$ is a parameter with dimensions of stress. Then letting ()$^{\prime}=\mathrm{d}() / \mathrm{d} \bar{z}$, dropping the bars for notational convenience, and referring equations to the initial configuration, the force balance equations in the meridional and normal directions are

$$
\begin{gathered}
T_{1}^{\prime}+\left(T_{1}-T_{2}\right) \eta / \lambda_{2}=0 \\
\kappa_{1} \sigma_{1} \lambda_{3}+\kappa_{2} \sigma_{2} \lambda_{3}=P
\end{gathered}
$$

where

$$
\kappa_{1}=\frac{\left(\eta \lambda_{1}^{\prime}-\lambda_{1} \eta^{\prime}\right)}{\lambda_{1}^{2}\left[\lambda_{1}^{2}-\eta^{2}\right]^{1 / 2}}, \quad \kappa_{2}=\frac{\left[\lambda_{1}^{2}-\eta^{2}\right]^{1 / 2}}{\lambda_{1} \lambda_{2}}
$$

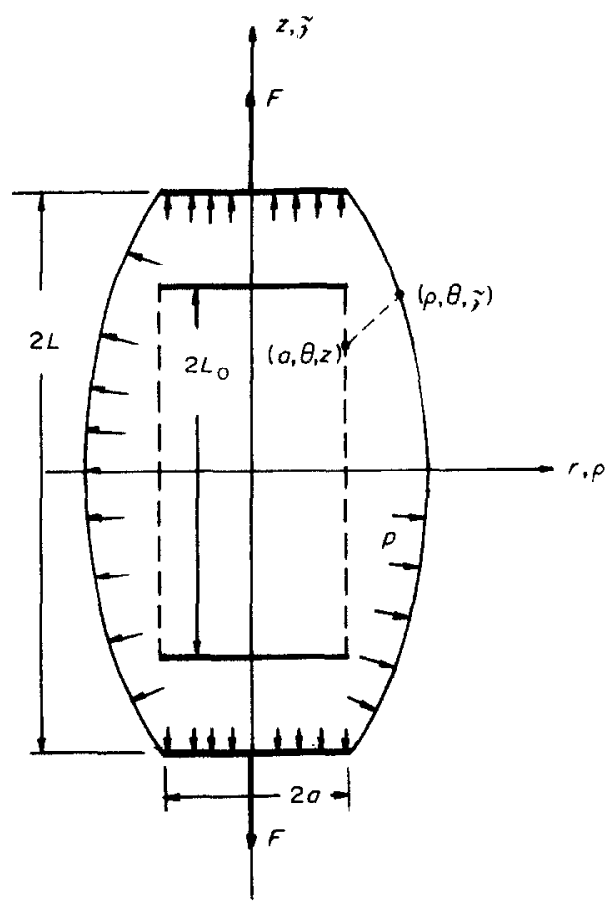

Fig. 1. Undeformed and deformed configurations of tubular membrane. Dashed lines denote undeformed configuration. 


$$
\eta=\lambda_{2}^{\prime}, \quad P=\frac{p a}{C_{0} h_{0}},
$$

and $p$ is the pressure.

For an incompressible nonlinear isotropic elastic membrane material, the constitutive equation is

$$
\sigma_{\alpha}=2\left(\lambda_{\alpha}{ }^{2}-\lambda_{3}{ }^{2}\right)\left(W_{1}+\lambda_{\beta}{ }^{2} W_{2}\right),
$$

where

$$
\begin{gathered}
W_{i}=\partial W / \partial I_{i}, \quad i=1,2, \\
I_{1}=\lambda_{1}{ }^{2}+\lambda_{2}{ }^{2}+\lambda_{3}{ }^{2}, \quad I_{2}=\lambda_{1}{ }^{-2}+\lambda_{2}{ }^{-2}+\lambda_{3}{ }^{-2},
\end{gathered}
$$

and

$$
\alpha=1, \quad \beta=2 \text { or } \alpha=2, \quad \beta=1 \text {. }
$$

For computational purposes, the strain energy function $W$ is taken as that given by Alexander[5]:

$$
\begin{gathered}
W_{1}=C_{1} \mathrm{e}^{k\left(I_{1}-3\right)^{2}}, \quad W_{2}=\frac{C_{2}}{\left(I_{2}-3\right)+\gamma}+C_{3}, \\
C_{1}=17.0, \quad C_{2}=19.85, \quad C_{3}=1.0, \quad \gamma=0.735 .
\end{gathered}
$$

The non-dimensionalizing parameter $C_{0}$ is taken as $C_{1}$.

Equations (2.1)-(2.4) can be reduced to a system of three simultaneous nonlinear ordinary differential equations for $\lambda_{1}, \lambda_{2}$ and the associated kinematic variable $\eta$ defined in (2.2c), (see [6]). This is achieved by substituting from (2.3) into (2.2a), and solving for $\lambda_{1}^{\prime}$ and $\eta^{\prime}$. The compatibility relation in $(2.2 \mathrm{c})$ completes the system. An alternate formulation which avoids redoing this algebra for each new choice of strain energy function $W$ was used instead. Defining

$$
\hat{\sigma}_{1}=\frac{\sigma_{1}}{\lambda_{1}}, \quad \hat{\sigma}_{2}=\frac{\sigma_{2}}{\lambda_{2}}, \quad \hat{\eta}=\frac{\eta}{\lambda_{1}}
$$

where the stresses are non-dimensional, (2.2) reduces to

$$
\begin{aligned}
& \hat{\sigma}_{1}^{\prime}=\hat{\sigma}_{2} \hat{\eta}, \\
& \lambda_{2}^{\prime}=\lambda_{1} \hat{\eta}, \\
& \hat{\eta}^{\prime}=\left(1-\hat{\eta}^{2}\right) \hat{\sigma}_{2} / \hat{\sigma}_{1}-P \lambda_{1} \lambda_{2}\left[1-\hat{\eta}^{2}\right]^{1 / 2} / \hat{\sigma}_{1} .
\end{aligned}
$$

Supplemented by (2.3), these are five equations for $\lambda_{1}, \lambda_{2}, \hat{\sigma}_{1}, \hat{\sigma}_{2}$ and $\hat{\eta}_{.} \hat{\sigma}_{2}$ is eliminated by using (2.3) with $\alpha=2$ and (2.5) to express it in terms of $\lambda_{1}$ and $\lambda_{2}$. Again, with $\alpha=1(2.3)$ and (2.5) give effectively

$$
\hat{\sigma}_{1}=\hat{\sigma}_{1}\left(\lambda_{1}, \lambda_{2}\right) \text {. }
$$

This is assumed invertible to give

$$
\lambda_{1}=g\left(\hat{\sigma}_{1}, \lambda_{2}\right) .
$$

An explicit inverse is not actually obtained. Equation (2.7) is inverted numerically, which is discussed in the next section. Using (2.8) reduces the system (2.6) to one in $\hat{\sigma}_{1}, \hat{\eta}$ and $\lambda_{2}$,

$$
\frac{\mathrm{d} \Lambda}{\mathrm{d} z}=F\left(\hat{\sigma}_{1}, \hat{\eta}, \lambda_{2} ; P\right),
$$


where

$$
\Lambda=\left(\hat{\sigma}_{1}, \hat{\eta}, \lambda_{2}\right)
$$

Let $F$ be the force applied to an end plate and $2 L$ be the prescribed deformed length. Following non-dimensionalization, $L_{0}$ becomes $L_{0} / a, L$ becomes $L / a$ and $F$ becomes $F /\left(\pi a h_{0} C_{0}\right)$. Appropriate boundary conditions are: by $(2.1),(2.2 \mathrm{c}),(2.5)$ and the symmetry of deformation about $z=0$,

$$
\hat{\eta}(0)=0 \text {, }
$$

and by the rigidity of the end plates,

$$
\lambda_{2}\left(L_{0}\right)=1
$$

(where $L_{0}$ denotes $L_{0} / a$ ).

These are joined by

$$
z\left(L_{0}\right)=\int_{0}^{L_{0}} \lambda_{1}\left[1-\hat{\eta}^{2}\right]^{1 / 2} \mathrm{~d} z=L
$$

if elongation is prescribed, or by

$$
F=\left.\left(2 \hat{\sigma}_{1}-P \lambda_{2}{ }^{2}\right)\right|_{z=0}
$$

if end force is prescribed.

The nonlinearity of (2.9) requires numerical integration. This proceeds from $z=0$ using (2.10a) and values for $\lambda_{2}(0)$ and $\hat{\sigma}_{1}(0)$. In the actual numerical procedure, values for $\lambda_{1}(0)$ and $\lambda_{2}(0)$ are selected and $\hat{\sigma}_{1}(0)$ is computed using (2.3) and (2.5). If elongation is prescribed, $\lambda_{1}(0)$ and $\lambda_{2}(0)$ are to be found so that (2.10b) and (2.11a) are met at $z=L_{0}$. If force is prescribed, $\lambda_{1}(0)$ and $\lambda_{2}(0)$ are to be found satisfying $(2.11 \mathrm{~b})$ at $z=0$ so that $(2.10 \mathrm{~b})$ is met at $z=L_{0}$.

The above formulations define two point boundary value problems. If elongation is prescribed a shooting method in two dimensions must be used. If force is prescribed, (2.11b) defines a curve in the $\lambda_{1}(0)-\lambda_{2}(0)$ plane, so that a shooting method in only one-dimension is needed.

The elongation boundary condition is specified for two reasons: (1) It is computationally more interesting, (2) elongation is more likely to be the controlling parameter in an experimental situation. The method of solution associated with this problem is discussed in the next section.

\section{NUMERICAL SOLUTION}

There are two subjects to be discussed. The first is the numerical integration of (2.9) subject to the inversion (2.8). The second is the method of satisfying boundary conditions.

The system of nonlinear ordinary differential equations (2.9) is integrated by a fourth order Runga-Kutta method. In this method, nodal values $\hat{\sigma}_{1}\left(z_{i}\right), \hat{\eta}\left(z_{i}\right)$ and $\lambda_{2}\left(z_{i}\right)$ are incremented and the r.h.s. of (2.9) is evaluated. Now, in the derivation of (2.9), an explicit expression (2.8) for the elimination of $\lambda_{1}$ is not actually obtained. Rather, for each new set of values of $\hat{\sigma}_{1}$ and $\lambda_{2},(2.7)$ is a nonlinear equation for $\lambda_{1}$. This is solved by iteration to yield, in effect, the evaluated inverse at required arguments.

Regarding the boundary conditions, the problem as defined in Section 2 requires finding $\lambda_{1}(0), \lambda_{2}(0)$ to satisfy two conditions specified at $z=L_{0}$, for prescribed pressure. A shooting method for solving this direct problem will be presented elsewhere. Here, in conjunction with the optimization scheme, the inverse approach is used. Values for $\lambda_{1}(0)$ and $\lambda_{2}(\theta)$ are specified and held fixed. Then end variables $\lambda_{2}\left(L_{0}\right)$ and $z\left(L_{0}\right)$ are functions of $P$. By $(2.10 \mathrm{~b}), P$ is chosen to satisfy

$$
\tilde{\lambda}(P)=\lambda_{2}\left(L_{0}\right)-1=0
$$


and $z\left(L_{0}\right)$ is taken as the corresponding calculated value for use in the optimizing procedure. Newton's method is used to solve the above for $P$.

\section{OPTIMIZATION}

As discussed in Sections 2 and 3, for a given pressure and elongation, the solution of the membrane problem can be obtained by integrating (2.9) with a correct set of initial data $\lambda_{1}(0)$ and $\lambda_{2}(0)$. This involves a two parameter shooting procedure which can be made reasonably efficient. Since we are only interested in the maximum pressure for a given elongation, the search for such a pressure could require repeated use of this procedure. The repeated use of even an efficient method can result in unacceptable accumulation of computing time and cost.

These considerations led to the selection of the projected gradient method in order to maximize the pressure and yet maintain computational efficiency. The core of this method is the inverse version of the tube problem presented at the end of Section 3. The remainder of this section presents the theory of the method.

Let a two dimensional space be defined by the cartesian coordinates $\left(\lambda_{1}(0), \lambda_{2}(0)\right)$. For notational convenience, this initial data space will be denoted as the $x-y$ space. Given a set of reasonable values for $(x, y)$, solution of the inverse problem gives a set of values for pressure and elongation. This implies the relations

$$
\begin{aligned}
& P=P(x, y), \\
& L=L(x, y),
\end{aligned}
$$

although these functions are evaluable only by computation.

The critical pressure problem can now be stated as the constrained optimization problem:

$$
\max P(x, y)
$$

$$
\text { subject to } L(x, y)==L_{f}
$$

where $L_{f}$ is a fixed length.

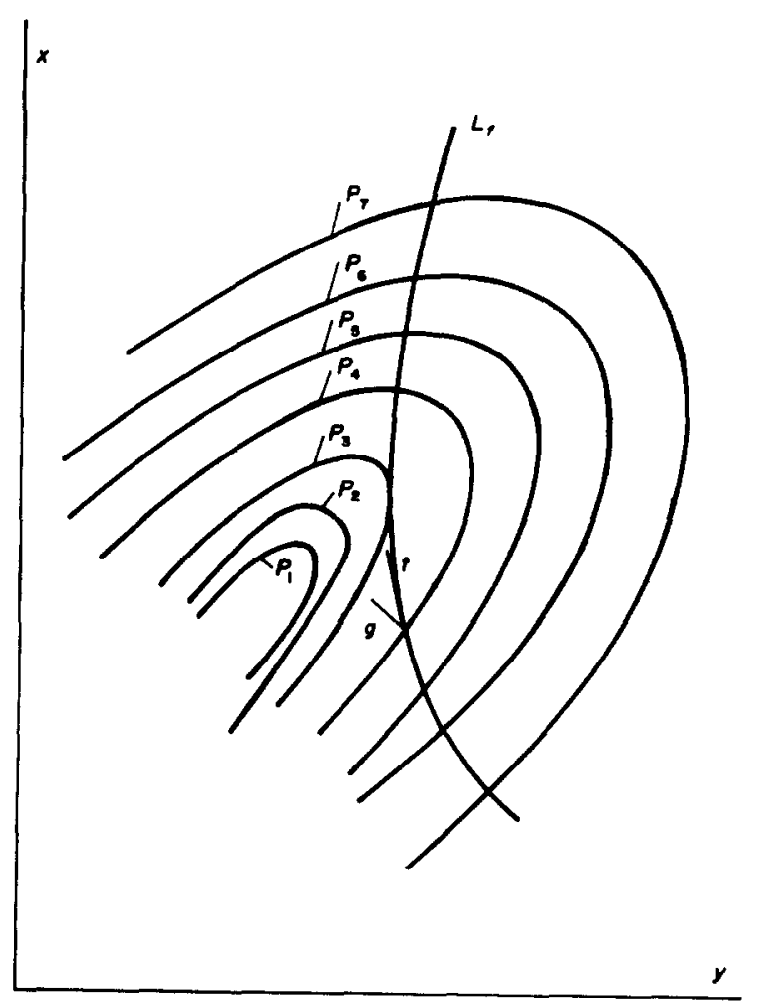

Fig. 2. Theoretical constraint curve $L_{f}$ and constant pressure contours. 
Observe that (4.1) defines two families of curves in the $x, y$ plane, shown in Fig. 2. The nested curves are the constant pressure contours $P(x, y)=P_{i}, P_{1}>P_{2}>\cdots>P_{n}$. Curve $L_{f}$ represents the constraint, which intersects the pressurc contours for some range of values of $P_{i}$. Moving along the constraint, the pressure increases to a maximum and then decreases. At the point of maximum pressure, the pressure contour is tangent to the constraint curve. There the pressure gradient $g=\nabla P$ is normal to the unit tangent $t$ to the constraint curve. The projected gradient method provides a systematic method for constructing a sequence of points along the constraint in the direction of increasing $P$ until $(g t)=0$.

We first find a point $\left(x_{1}, y_{1}\right)$ that satisfies $L\left(x_{1}, y_{1}\right)=L_{f}$. A move along the direction of the gradient, $g=\nabla P$, to a neighboring point $\left(x^{*}, y^{*}\right)$ will increase the value of $P$, but may not satisfy $L\left(x^{*}, y^{*}\right)=L_{f}$. In order to increase the value of $P$ and still satisfy the constraint condition, we need to move along the tangent direction to the curve $L(x, y)=L_{f}$ in the sense of positive gradient projection.

Let $t$ be the unit tangent vector at a point $\left(x_{n}, y_{n}\right)$ of the constraint curve $L(x, y)=L_{f}$. The projected gradient vector is given by

$$
g^{t}=(g t) t
$$

Determination of the next point $\left(x_{n+1}, y_{n+1}\right)$ on the constraint curve in the direction of increasing $P$ is done in two stages. The first stage is to determine a point along the projected gradient vector

$$
\left(\bar{x}_{n+1}, \bar{y}_{n+1}\right)=\left(x_{n}, y_{n}\right)+k g^{\prime}\left(x_{n}, y_{n}\right), \quad(n=1,2, \ldots)
$$

where $k$ is a positive number. The second stage is to backtrack to the constraint curve. The method of selecting $k$ in the numerical example will be discussed in more detail in Section 5 .

To backtrack to the constraint curve, a one-dimensional search is carried out along either the line $x_{n+1}=\tilde{x}_{n+1}$ or $y_{n+1}=\tilde{y}_{n+1}$. The complete step gives a new point $\left(x_{n+1}, y_{n+1}\right)$ such that

$$
\begin{gathered}
P\left(x_{n+1}, y_{n+1}\right)>P\left(x_{n}, y_{n}\right), \\
L\left(x_{n+1}, y_{n+1}\right)=L_{f} .
\end{gathered}
$$

The critical pressure is reached when $(g t)=0$, or computationally when $(g t)$ changes sign. Computational details are presented in the next section.

\section{COMPUTATIONAL DETAILS}

As the first steps in the projected gradient method, an initial starting point $\left(x_{0}^{\prime}, y_{0}\right)$ is assumed, $P$ satisfying (3.1) is calculated and $L$ is evaluated. A one-dinensional search in $x$, using Newton's method, gives a point $\left(x_{0}, y_{0}\right)$ on the constraint curve by solving

$$
\left|L\left(x, y_{0}\right)-L_{f}\right|<\epsilon_{1}
$$

The initial increment in $x$ was typically 0.01 and $\epsilon_{1} \approx 10^{-4}$.

Once a point $\left(x_{1}, y_{1}\right)$ on the constraint curve has been found, approximations to vectors $g$ and $t$ are constructed. First, $P$ and $L$ are computed at $\left(x_{1}, \hat{y}_{1}\right)$, where $\hat{y}_{1}=y_{1}+\Delta y$. As $L\left(x_{1}, \hat{y}_{1}\right) \neq L_{f}$ in general, another one-dimensional search in $x$ gives a point $\left(\hat{x}_{1}, \hat{y}_{1}\right)$ on the constraint curve, i.e.

$$
\left|L\left(\hat{x}_{1}, \hat{y}_{1}\right)-L_{f}\right|<\epsilon_{1}
$$

An approximation to the unit tangent can be constructed from the points $\left(x_{1}, y_{1}\right)$ and $\left(\hat{x}_{1}, \hat{y}_{1}\right)$ on the constraint curve. Values for $P$ have been computed at the three points $\left(x_{1}, y_{1}\right),\left(x_{1}, \hat{y}_{1}\right)$ and $\left(\hat{x}_{1}, \hat{y}_{1}\right)$. An approximation to the gradient component $\partial P / \partial y$ can be constructed by finite differences from values at $\left(x_{1}, y_{1}\right)$ and $\left(x_{1}, \hat{y}_{1}\right)$. The component $\partial P / \partial x$ can be similarly found from values at $\left(x_{1}, \hat{y}_{1}\right)$ and $\left(\hat{x}_{1}, \hat{y}_{1}\right)$. 
To obtain the next point in the sequence defined by (4.4), a step in the tangent direction of $k \operatorname{sgn}(g t)$ is taken. The whole process of backtracking, construction of $g$ and $t$, and stepping is then repeated. When ( $g t$ ) changes sign, $\Delta y$ and $k$ are replaced by $\mu \Delta y$ and $\mu k$, where $\mu<1$, to cut down the step size. The process is then repeated. In this manner, the position of the local maximum can be determined within an interval of length $k^{\prime}$ on the constraint curve, where $k^{\prime}$ is the current reduced value of $k$. The alternate approach of using the condition $|(g t)|<\epsilon$ was abandoned because the constant pressure and constraint curves were found to be nearly tangent over a long interval. Initial values for $k$ can be chosen somewhat arbitrarily. Results were obtained with $k=0.1$. In addition $\mu=0.1$, and the search terminates when $k^{\prime}=10^{-3}$.

Except as noted below, this procedure was insensitive to the initial estimate $\left(x_{0}, y_{0}\right)$. The pressure determined for each point $(x, y)$ was taken as the first estimate in the search for the pressure at the next point. This worked very well, allowing the correct pressure to be found after an average of 12 iterations. The one-dimensional searches also converged rapidly, generally in less than four iterations.

Some numerical difficulties were encountered in the numerical integration of the system of differential equations (2.9). For small pressures and elongations $\left(P \approx 0, L \approx L_{0}\right)$, the initial pressure estimate had to be very close to the converged value, or the argument of the square root in (2.6) became negative. A similar problem arose near the local maximum. In this latter case, search steps were taken into a region where, for given $x, y$ and $L_{f}$, no physical solution was possible. Reducing $\Delta y$ and $k$ by a factor of 10 , and resuming computation at the last successful point allowed the maximum point to be found.

In the numerical example, the initial half length to radius ratio $L_{0}$ was taken as 1 . The largest integration step was $\Delta z=0.1$. Condition (3.1) was considered satisfied when $\tilde{\lambda}<10^{-4}$.

Using results obtained during computation, it was possible to construct the two families of

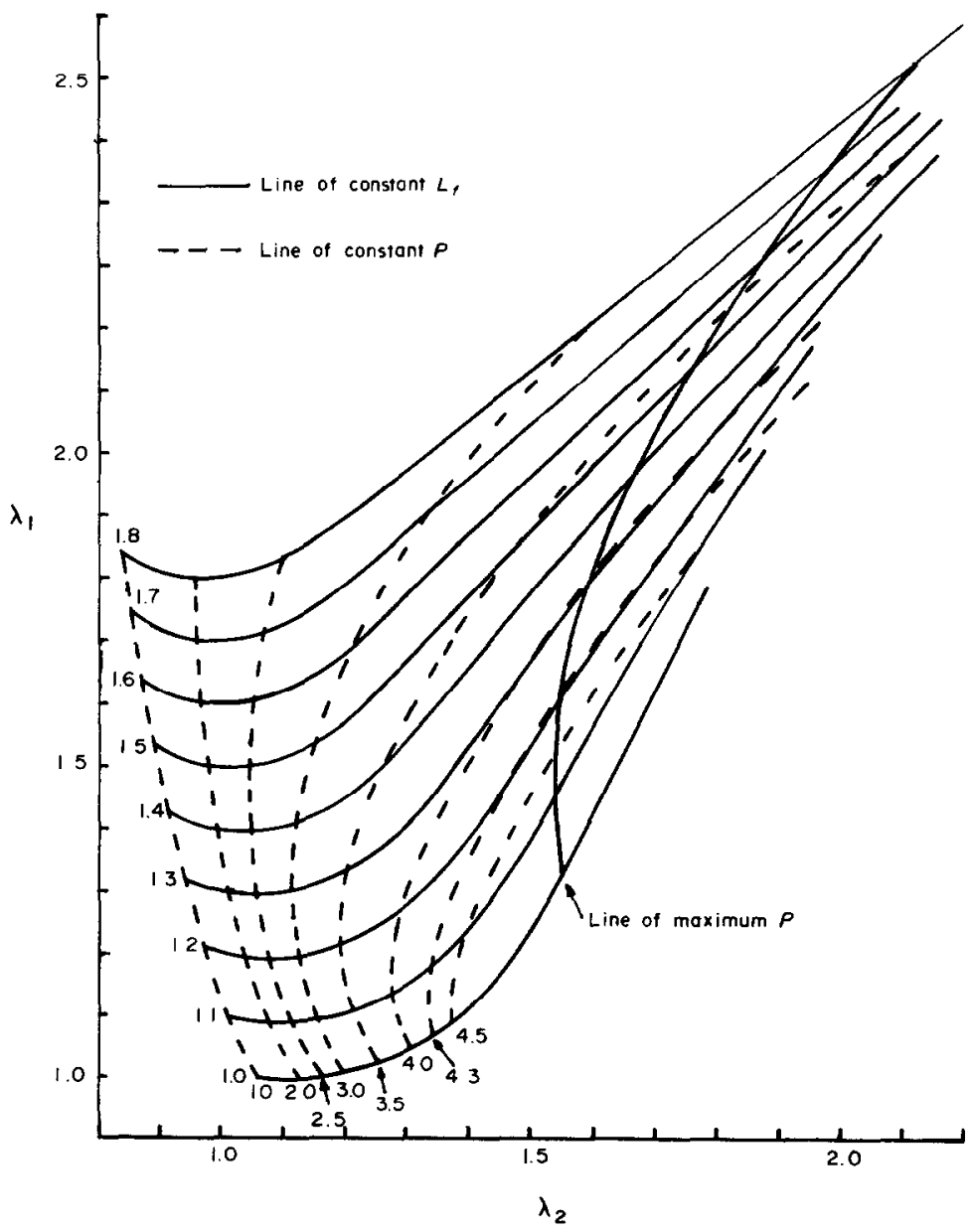

Fig. 3. Computed constraint curve and constant pressure contours. 
curves defined by (4.1). These are shown in Fig. 3, where solid lines denote the constraint curve $L(x, y)=L_{f}$ and dashed lines denote the constant pressure curve $P(x, y)$. The heavy solid line passes through the local pressure on each constraint curve determined when $k^{\prime}<\epsilon_{2}$. In order to emphasize this locus of local maxima, points were computed on the constraint curves in the range of monotonically decreasing pressure. These were obtained by changing the sign of the pressure gradient in the search program and restarting computations just past the local maximum point.

Figure 3 provides an interesting display of the pressure-elongation interaction within the range of values shown. First note the major result of this work, that for the strain energy function considered there is indeed a local maximum pressure for each prescribed elongation. Figure 4 shows local maximum pressure vs tube elongation. As the tube is elongated the local

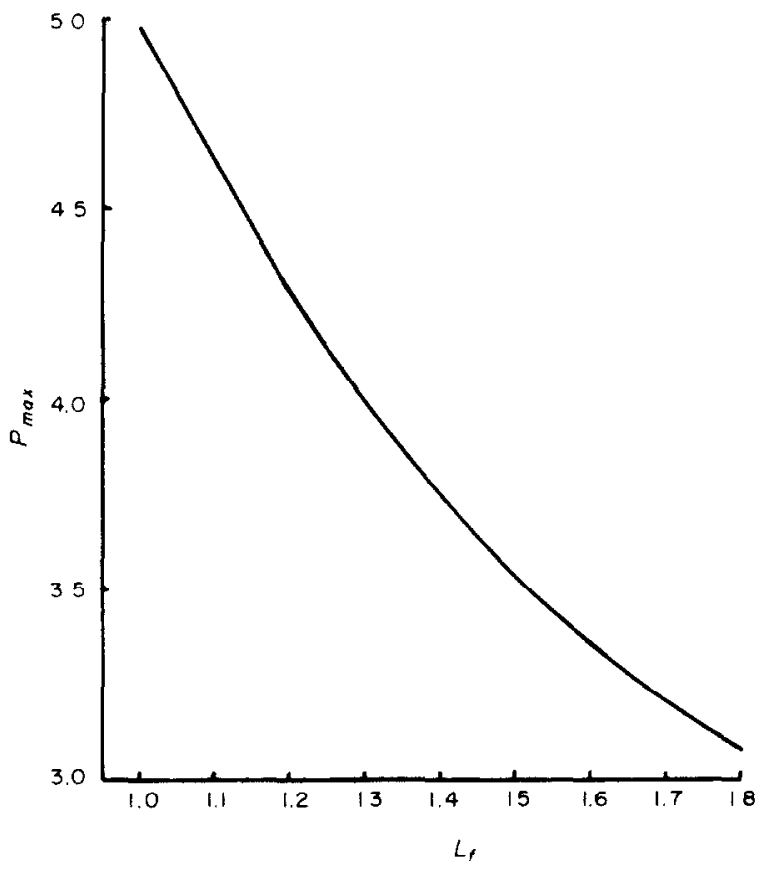

Fig. 4. Limiting pressure for prescribed elongation.

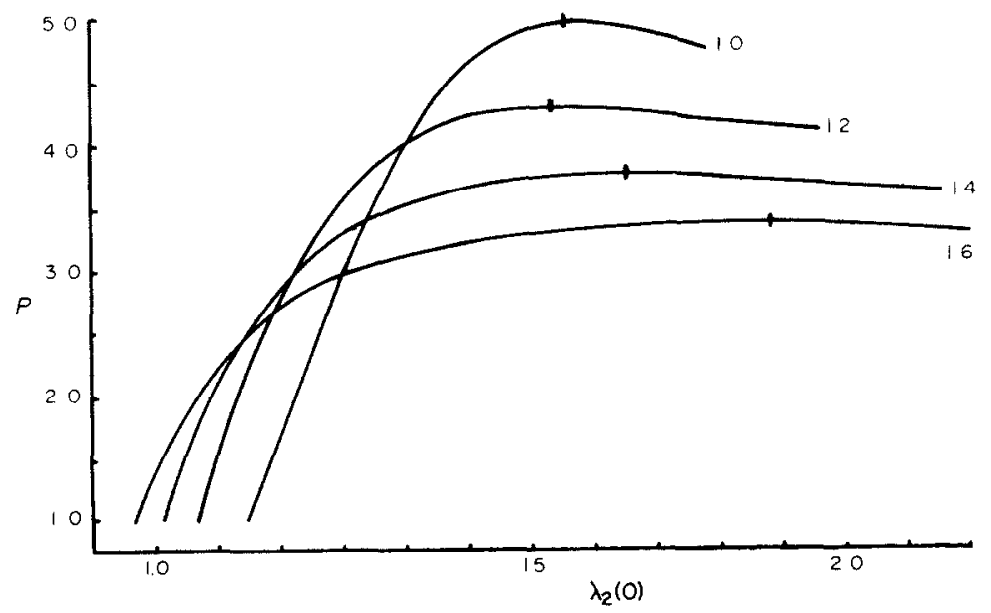

Fig. 5. Pressure vs deformed radius at midlength $\rho(0) / a$ for various elongations. 
maximum pressure decreases and occurs at larger values of $\lambda_{1}(0)$ and $\lambda_{2}(0)$. Figure 5 shows pressure vs deformed radius at the membrane center for several prescribed elongations.

The relation between the family of curves in Fig. 3 is consistent with that shown in Fig, 2. The curvature of the constant pressure curves are opposite those at the constraint curves. Furthermore, each constant pressure curve intersects constraint curves for only a limited range of elongation. Each constraint curve is tangent to a constant pressure curve at its local maximum pressure. Constant pressure curves for lower constant pressure intersects the constraint curves in a second location which is in the decreasing pressure range. Beyond the local maximum, in the region of larger values of $\lambda_{1}(0)$ and $\lambda_{2}(0)$, the constraint curves appear to approach straight lines. A detailed study of this behavior may be possible through an asymptotic analysis of $(2.6)$ as $\lambda_{1}(0)$ and $\lambda_{2}(0)$ become large, but is beyond the scope of this work.

Acknowledgement-The authors are grateful to the National Science Foundation for support of this work under Grant No. ENG75-17489.

\section{REFERENCES}

1. A. E. Green and R. T. Shield, Proc. R. Soc. A202, 407 (1950).

2. J. E. Adkins and R. S. Rivlin, Large elastic deformations of isotropic materials-IX. Phil. Trans. R. Soc. A244, 505 (1952).

3. A. D. Kydoniefs and A. J. M. Spencer, The finite inflation of an elastic toroidal membrane of circlar cross section. Int. J. Engng Sci. 5, 367 (1967).

4. A. D. Kydoniefs and A. J. M. Spencer, Finite axisymmetric deformation of an initially cylindrical elastic membrane. $Q$. J. Mech. Appl. Math. 22, 87 (1999).

5. H. Alexander, A constitutive relation for rubberlike materials. Int. J. Engng Sci. 6. 549 (1968).

6. A. S. Wineman, On the axisymmetric inflation of nonlinear viscoelastic membranes. Proc. 6th Canadian Cong. of Applied Mechanics, Vancouver, p. 41 (1977). 